\title{
Ultrafast excited state absorption and charge separation in phenylene-based conjugated polymers
}

\author{
Alok Shukla ${ }^{\mathrm{a}}$, Haranath Ghosh ${ }^{\mathrm{b}}$, Sumit Mazumdar ${ }^{\mathrm{c}, *}$ \\ a Indian Institute of Technology, Powai, Mumbai 400076, India \\ ${ }^{\mathrm{b}}$ Centre for Advanced Technology, Indore 452013, India \\ ${ }^{c}$ Department of Physics, University of Arizona, Tucson, AZ 85721, USA
}

\begin{abstract}
Ultrafast pump-probe spectroscopy of phenylene-based $\pi$-conjugated polymers give low and high energy photoinduced absorptions (PAs) to final states with very different relaxation behavior. While the low energy two-photon state that is reached in PA relaxes to the optical exciton in ultrafast times, the higher energy two-photon state violates the Vavilov-Kasha rule and instead of relaxing to the optical exciton undergoes interchain dissociation to a polaron pair state. We have calculated the excited state absorptions of long oligomers of poly-paraphenylene and poly-paraphenylenevinylene within a rigid band correlated electron Hamiltonian, using the complete $\pi$-electron basis and the powerful multi-reference singles and double configuration interaction technique. Our calculated PA spectra well reproduce the experimental PA. We further show that there exists a fundamental difference in the natures of the wavefunctions of the final states that are reached in low and high energy PA, and the difference originates from the existence of multiple kinds of bands in phenylene-based systems within one-electron theory. We speculate that the violation of the Vavilov-Kasha rule and charge separation into polaron pairs upon excitation to the high energy two-photon state is a consequence of its specific electronic structure, and discuss an earlier work on wavelength dependent photoconductivity of poly-paraphenylenevinylene to support this conjecture.
\end{abstract}

PACS: 78.47.+p; 78.20.Bh; 42.65.Re; 78.40.Me; 78.66.Qn

Keywords: Photoinduced absorption; Poly( $p$-phenylene); Poly( $p$-phenylenevinylene); Charge separation

\section{Introduction}

To harness the full potential of $\pi$-conjugated polymers, it is important to reach complete understanding of their excited states and their roles in the photophysics of these systems. One-photon $\mathrm{B}_{\mathrm{u}}$ excited states are explored through linear spectroscopy and are by now well understood, even for complicated phenylene-based $\pi$-conjugated polymers [1-4]. Two-photon $A_{g}$ excited states can be probed only by nonlinear spectroscopic techniques such as photoinduced absorption (PA) [5-13], electroabsorption (EA) [7,14,15], third-harmonic generation (THG) [16], and two-photon absorption (TPA) $[9,17,18]$. Frolov et al. [8,9] discovered the existence of two prominent PA bands in PPV derivatives, labeled hereafter as low energy PA1 $(\sim 0.8 \mathrm{eV})$ and high energy PA2 $(\sim 1.3-1.4 \mathrm{eV})$, respectively. The $\mathrm{A}_{\mathrm{g}}$ state that gave rise to PA1 also contributes strongly to EA [14] and TPA [9], and therefore, in analogy with the linear chain polyacetylenes and polydiacetylenes, where a specific $\mathrm{mA}_{\mathrm{g}}$ ( $\mathrm{m}$ being a unknown chain-length and Coulomb correlation dependent quantum number) excitonic state overwhelmingly dominates third order optical nonlinearity [19-26], Frolov et al. ascribed PA1 to excited state absorption to the $\mathrm{mA}_{\mathrm{g}}$ state of PPV derivatives. PA2 was ascribed to a hitherto unknown $\mathrm{kA}_{\mathrm{g}}$ state, which has no counterpart in the linear chain polymers [14]. The distinguishing feature of the $\mathrm{kA}_{\mathrm{g}}$ state is its relaxation mechanism. While the $\mathrm{mA}_{\mathrm{g}}$ state relaxes to the $1 \mathrm{~B}_{\mathrm{u}}$ at ultrafast times, the $\mathrm{kA}_{\mathrm{g}}$ dissociates into an interchain polaron pair [9]. This behavior of the $\mathrm{kA}_{\mathrm{g}}$ is in strong violation of the Vavilov-Kasha rule which asserts that all excited states of a given spin symmetry relax to the lowest state within the same spin subspace by means of internal conversion [27]. Other investigators have also observed similar dissociation to polaron pair from high energy two-photon states in PPV derivatives, polyfluorene, ladder-type PPP and its oligomers [10-13]. 
The unusual relaxation behavior of the $\mathrm{kA}_{\mathrm{g}}$ is an intriguing phenomenon that raises the following important question: is the efficient interchain charge-transfer from the $\mathrm{kAg}_{\mathrm{g}}$ merely a consequence of the high energy of this state, or is this a signature of different physical character of the $\mathrm{kAg}_{\mathrm{g}}$ wavefunction? In an earlier work based on theoretical study of two-photon states of biphenyl and triphenyl within a restricted basis space, Chakrabarti and Mazumdar [28] had indeed posited the occurrence of multiple classes of two-photon states in phenylene-based $\pi$-conjugated polymers. This work, however, was far from conclusive, both because of the very short lengths of the molecules studied, and because all molecular orbitals (MOs) below and above the localized benzene bonding and antibonding MOs were ignored in the configuration interaction (CI) calculations [28]. In the light of the recent experiments [9-13], we revisit the problem of two-photon states and PA in PPP and PPV and perform correlated electron calculations for relatively long oligomers. Our aim is not just to classify the two-photon states based upon their many-body wave functions, but also to arrive at a plausible explanation for the efficient interchain charge-transfer from the $\mathrm{kAg}_{\mathrm{g}}$.

In the present study, we have performed multireference singles doubles configuration interaction (MRSDCI) calculations within the pariser-parr-pople (P-P-P) model Hamiltonian for oligomers containing up to five phenylene units for PPP and four phenylene units for PPV, using the full $\pi$-electron basis space. We shall refer to these oligomers with $n$-phenyl units as PPPn and PPVn, respectively. We obtain, and diagonalize very large CI matrices, containing in some cases close to a million configurations. To the best of our knowledge, the present calculations are the most accurate correlated electron calculations that incorporate the full basis space for the polyphenylenes. We find clear evidence that the natures of the configurations that dominate the $\mathrm{mA}_{\mathrm{g}}$ and the $\mathrm{kAg}_{\mathrm{g}}$ are qualitatively different. Both the singly and doubly excited configurations that dominate the $\mathrm{mA}_{\mathrm{g}}$ involve mostly the inner delocalized bonding and antibonding MOs that occur above and below the localized benzene MOs. In contrast, the singly excited configurations that dominate the $\mathrm{kA}_{\mathrm{g}}$ involve also the outer delocalized MOs, while the dominant doubly excited configurations involve both delocalized and localized MOs. We posit that it is this qualitatively different nature of the $\mathrm{kA}_{\mathrm{g}}$ that is responsible for its efficient dissociation, and argue that an earlier one-photon optical and photoconductivity study of PPV [29] provide indirect support of the mechanism of two-photon induced charge-transfer suggested here.

The remainder of the paper is organized as follows. In the next section, the methodology underlying our calculations is discussed. This is followed by a brief review of the theory of ground-state absorptions in PPV in Section 3. The purpose of this section is to point out that based on this theory, which shows the existence of multiple classes of one-photon states, the occurrence of multiple clases of two-photon states is perhaps to be anticipated. In Section 4, we present and discuss the results of our calculations of PA and the wavefunctions of the different classes of two-photon states. Finally in Section 5 we discuss the main conclusions of our work, with emphasis on comparison to experiments.

\section{The theoretical model and methodology}

We consider oligomers of PPP and PPV within the P-P-P model Hamiltonian

$$
\begin{aligned}
H= & -\sum_{\langle i j\rangle, \sigma} t_{i j}\left(c_{i \sigma}^{\dagger} c_{j \sigma}+c_{j \sigma}^{\dagger} c_{i \sigma}\right)+U \sum_{i} n_{i \uparrow} n_{i \downarrow} \\
& +\sum_{i<j} V_{i j}\left(n_{i}-1\right)\left(n_{j}-1\right)
\end{aligned}
$$

where $\langle i j\rangle$ implies nearest neighbors, $c_{i \sigma}^{\dagger}$ creates a $\pi$-electron of spin $\sigma$ on the $p_{z}$ orbital of carbon atom $i, n_{i \sigma}=c_{i \sigma}^{\dagger} c_{i \sigma}$ is the number of electrons with spin $\sigma$, and $n_{i}=\sum_{\sigma} n_{i \sigma}$ is the total number of $\pi$-electrons on atom $i . U$ and $V_{i j}$ are the on-site and long-range Coulomb interactions, respectively, while $t_{i j}$ is the nearest neighbor one-electron hopping matrix element. Our parametrization of the $V_{i j}$ is similar to the Ohno parametrization [30]

$$
V_{i j}=\frac{U}{\kappa\left(1+0.6117 R_{i j}^{2}\right)^{1 / 2}}
$$

where $\kappa$ is a parameter which screens the intersite Coulomb interactions [1,31]. We have examined both the standard Ohno parameters $(U=11.13 \mathrm{eV}, \kappa=1.0)$, as well as a particular combination of $U$ and $\kappa(U=8.0 \mathrm{eV}, \kappa=2.0)$ that was shown previously to be satisfactory at a semiquantitative level for explaining the full wavelength dependent ground state absorption spectrum of PPV [1]. We shall hereafter refer to this second set of parameters as screened Ohno parameters. As far as the hopping matrix elements are concerned, we took $t=-2.4 \mathrm{eV}$ for the $\mathrm{C}-\mathrm{C}$ bond in benzene rings. The hopping corresponding to the interbenzene single bond in the PPP oligomers was taken to be $t=-2.23 \mathrm{eV}$. For the vinylene linkage of the PPV oligomers, we chose the hopping elements to be $-2.2 \mathrm{eV}$ for the single bond, and $-2.6 \mathrm{eV}$ for the double bond. We considered planar PPP oligomers with $\mathrm{D}_{2} \mathrm{~h}$ symmetry, and planar PPV oligomers with $\mathrm{C}_{2} \mathrm{~h}$ symmetry, with the line joining the para-carbon atoms of the same phenyl group defined as the $x$-axis in both cases. The one-photon states belong to the $\mathrm{B}_{\mathrm{u}}$ irrep for oligo-PPV, and $\mathrm{B}_{1 u}$ ( $x$-polarized), and $\mathrm{B}_{2 u}$ (y-polarized) irreps for the oligo-PPPs. Since here we are concerned with excited state absorption from the lowest one-photon states, which are $x$-polarized in both cases, we will refer to the optical $\mathrm{B}_{1 u}$ state of PPP also as the $1 \mathrm{~B}_{\mathrm{u}}$ state for simplicity. Furthermore, experiments indicate that the observed PA are polarized along the $x$-direction (predominantly $x$-direction in PPV) [32], and hence we calculate only $x$-polarized PA 
Table 1

The number of reference configurations $\left(N_{\text {ref }}\right)$ and the total number of configurations ( $\left.N_{\text {total }}\right)$ involved in the MRSDCI (or QCI, where indicated) calculations, for different symmetry subspaces of the various oligomers

\begin{tabular}{lcccrr}
\hline Oligomer & \multicolumn{2}{c}{$\mathrm{A}_{\mathrm{g}}$} & & \multicolumn{2}{c}{$\mathrm{B}_{\mathrm{u}}$} \\
\cline { 2 - 3 } \cline { 5 - 6 } & $N_{\text {ref }}$ & $N_{\text {total }}$ & & $N_{\text {ref }}$ & $N_{\text {total }}$ \\
\hline PPP3 & $1^{\mathrm{a}}$ & 193678 & $1^{\mathrm{a}}$ & 335545 \\
PPP4 & 55 & 284988 & 15 & 76795 \\
PPP5 & 48 & 663619 & 7 & 87146 \\
PPV3 & 37 & 215898 & 12 & 220905 \\
PPV4 & 39 & 981355 & 3 & 225970 \\
\hline
\end{tabular}

${ }^{\mathrm{a}}$ QCI method.

from the $1 \mathrm{~B}_{\mathrm{u}}$ exciton to the $\mathrm{A}_{\mathrm{g}}$ states. Weak $y$-polarized PA to $\mathrm{B}_{\mathrm{g}}$ states are therefore ignored.

The correlated electron calculations were done using the MRSDCI approach, which is a powerful CI technique that was used previously for conjugated polymers by other investigators $[24,33]$, and by us to calculate the excited state ordering in polyphenyl- and polydiphenylacetylenes [34], and triplet PA in PPV [35]. We use very stringent convergence criterion for all excited states and the technical details of our MRSDCI methodology can be found in our earlier works $[34,35]$. However, to give some idea of the highly correlated nature of the wavefunctions of the excited states, in Table 1 we have listed the number of reference configurations that were used for each symmetry subspace of PPP3, PPP4 and PPP5, respectively, as well as the overall sizes of the Hamiltonian matrix in each case (note that for PPP3 the method used was quadruple-CI or QCI rather than MRSDCI). The number of MRSDCI reference functions are larger in the $\mathrm{A}_{\mathrm{g}}$ subspaces than in the $B_{u}$ subspace because while only the $1 B_{u}$ was optimized in the $B_{u}$ subspace, many different $A_{g}$ states (all those with significant transition dipole couplings with the $1 \mathrm{~B}_{\mathrm{u}}$ ) had to be simultaneously optimized in the $A_{g}$ subspace. All those configurations were included in the reference list whose coefficients were at least 0.1 in magnitude in any one of the targeted states. The $N_{\text {ref }}$ in Table 1 should be compared to the few (usually 2 or 3 ) reference states that are retained in calculations of the lowest $A_{g}$ states [33].

\section{Theory of ground state absorption and its implication}

Before we present our calculations of PA, it is useful to recall the results of calculations of the ground state absorption [1-4]. This is because multiple classes of final states are relevant also in ground state absorption, and as we indicate below analysis of the ground state absorption strongly suggests that there should occur multiple classes of two-photon states.

The band structure of PPP computed using the Hückel model is presented in Fig. 1. The bands labeled $d_{1}$ and $d_{1}^{*}$, and $d_{2}$ and $d_{2}^{*}$, are delocalized over all carbon atoms.

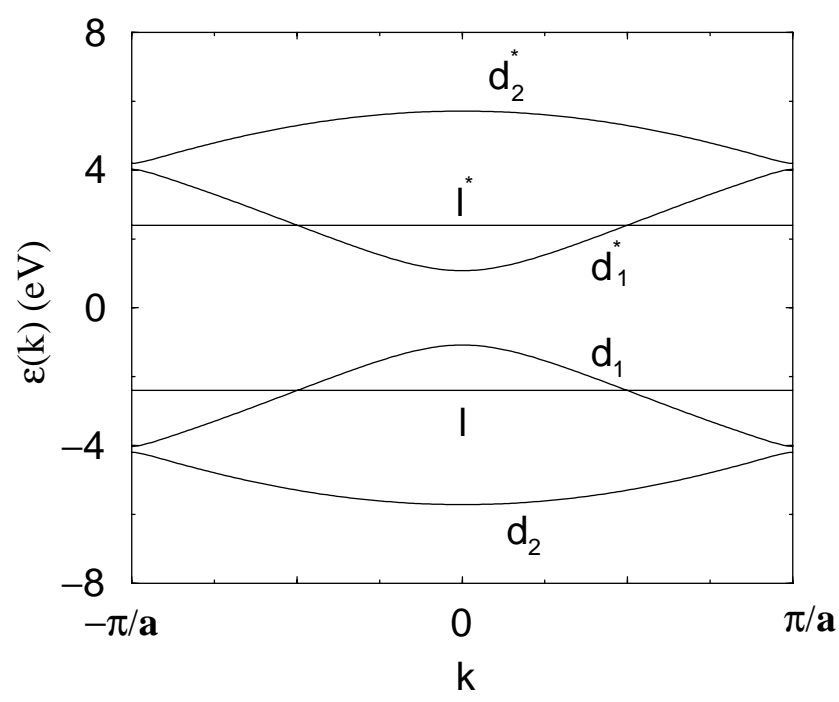

Fig. 1. Band structure of PPP computed using the Hückel model. Label next to each band points out the nature of the orbital associated with it.

The dispersionless $l$ and $l^{*}$ bands are localized on individual phenyl groups, with zero charge densities on the carbon atoms at $(1,4)$ positions. The band structure of PPV is qualitatively similar to that of PPP [36], with an additional pair of delocalized bands ( $d_{3}$ and $d_{3}^{*}$ ) further removed from the chemical potential.

Within band theory the ground state absorption consists of single excitations that are low energy $d_{1} \rightarrow d_{1}^{*}$, high energy $l \rightarrow l^{*}$, and intermediate energy $d_{1} \rightarrow l^{*}$ and $l \rightarrow d_{1}^{*}$, with the intermediate energy absorption band occurring exactly half way in between the low and high energy absorption band. Here we have ignored absorptions involving $d_{2}, d_{2}^{*}$, etc. bands, as excitations involving these bands lie outside the range of experimental wavelengths. The $d_{1} \rightarrow d_{1}^{*}$ and $l \rightarrow l^{*}$ bands are polarized along the $x$-direction in PPP and predominantly along the $x$-direction in PPV, while the $d_{1} \rightarrow$ $l^{*}$ and $l \rightarrow d_{1}^{*}$ bands are polarized along the $y$-direction and predominantly along the $y$-direction, respectively. Experimentally in PPV there occur absorptions at $\sim 2.4,3.7$, 4.7 and $\sim 6.0 \mathrm{eV}$, respectively. These have been explained within a correlated electron picture: the absorptions at 2.4 and $6.0 \mathrm{eV}$ are due to $d_{1} \rightarrow d_{1}^{*}$ and $l \rightarrow l^{*}$ exciton states, respectively [1-4]; the absorption at $3.7 \mathrm{eV}$ is to a higher energy $d_{1} \rightarrow d_{1}^{*}$ exciton [1]; and finally, the absorption at $4.7 \mathrm{eV}$ is to the "plus" linear combination of the excitations $d_{1} \rightarrow l^{*}+l \rightarrow d_{1}^{*}[1-4]$. The corresponding "minus" linear combination, $d_{1} \rightarrow l^{*}-l \rightarrow d_{1}^{*}$, occurs also at about $3.7 \mathrm{eV}$ $[1,2]$, but is forbidden in linear absorption. All of these assignments have been confirmed by polarization studies of absorptions in stretch-oriented samples $[1,4,37]$.

The relevance of these known results to the present case are as follows. Energetically, the $\mathrm{kA}_{\mathrm{g}}$ in PPV is at $\sim 3.6-3.8 \mathrm{eV}[8,9]$. Since ground state absorption occurs to a high energy $d_{1} \rightarrow d_{1}^{*}$ excitation of $\mathrm{B}_{\mathrm{u}}$ symmetry in this energy region, in principle, the $\mathrm{kA}_{\mathrm{g}}$ can simply be a 
similar $d_{1} \rightarrow d_{1}^{*}$ excitation of $\mathrm{A}_{\mathrm{g}}$ symmetry. If this were true, the $\mathrm{mA}_{\mathrm{g}}$ and the $\mathrm{kA}_{\mathrm{g}}$ would be qualitatively similar, and charge carrier creation from the $\mathrm{kA}_{\mathrm{g}}$ (but not from the $\mathrm{A}_{\mathrm{g}}$ ) can only be a consequence of possibly greater $\mathrm{e}-\mathrm{h}$ separation in the $\mathrm{kA}_{\mathrm{g}}$. An alternate possibility is that the $\mathrm{kA}_{\mathrm{g}}$ is dominated by configurations that are fundamentally different. Recall that the occurrence of the $2 \mathrm{~A}_{\mathrm{g}}$ below the $1 B_{u}$ in the polyacetylenes and polydiacetylenes is a general many-body phenomenon: $A_{g}$ eigenstates having strong double-excitation contributions from specific MOs can be close in energy, or can even occur below $B_{u}$ eigenstates that are dominated by single excitations involving the same MOs. Since the lowest energy single excitations involving the $d_{1} \rightarrow l^{*}$ and $l \rightarrow d_{1}^{*}$ excitations (the minus linear combinations mentioned above) occur at $\sim 3.7 \mathrm{eV}[1,2]$, it is to be anticipated that many-body $\mathrm{A}_{\mathrm{g}}$ eigenstates that are dominated by double excitations of the type $\left(d_{1} \rightarrow l^{*} ; l \rightarrow d_{1}^{*}\right)$ occur also within the same energy range. In principle then, the $\mathrm{kA}_{\mathrm{g}}$ can also be dominated by two-excitation components involving the $l$ and $l^{*}$ bands, and be qualitatively different from the $\mathrm{mA}_{\mathrm{g}}$. As shown in the following sections, we do indeed find evidence for such $\mathrm{A}_{\mathrm{g}}$ states with significant dipole coupling to the $1 \mathrm{~B}_{\mathrm{u}}$.

\section{Correlated electron calculations of PA spectra}

First we comment briefly on the proper choice of the Coulomb parameters for the P-P-P Hamiltonian (Eq. (1)). Our computed PA spectra of various PPP and PPV oligomers using the bare Ohno parameters were remarkably different from the experimentally observed spectra in all cases; specifically, the calculated PA1 intensity was smaller than the PA2 intensity [38], which is exactly opposite to what is observed experimentally. We therefore conclude that the bare Ohno parameters are not suitable for high energy states of PPP and PPV [38]. The same conclusion was arrived at earlier from calculations of ground state absorption in PPV [1] (see also [31]).

In Fig. 2 we have shown the calculated excited state absorptions for (a) PPP4; (b) PPP5; (c) PPV3; and (d) PPV4 for $U=8.0 \mathrm{eV}$ and $\kappa=2$. In all cases our abcissa is the energy scaled with respect to $E\left(1 \mathrm{~B}_{\mathrm{u}}\right)$. As pointed out before [39], convergence in the scaled energies of high energy states with increasing chain length is not expected in this region of relatively short chain lengths. This is because the lowest energy excitations (for example, the $1 \mathrm{~B}_{\mathrm{u}}$ and the $2 \mathrm{~A}_{\mathrm{g}}$ ) converge with increasing chain length much faster than the higher energy states. Taken together with the discrete nature of the excited states, this can indicate an apparent increase in the scaled energy of the high energy excited state with increasing chain length, even as the actual energy is decreasing. This is exactly what happens between PPP4 and PPP5, and between PPV3 and PPV4 in Fig. 2. Quantitative comparisons of the scaled energies of the theoretical excited state absorption bands and those of the

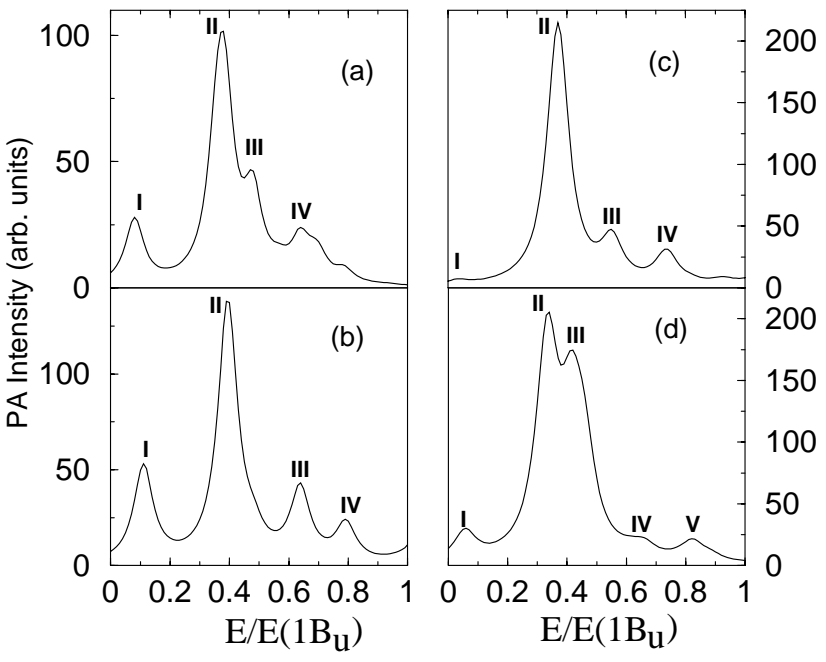

Fig. 2. Calculated PA spectra of (a) PPP4; (b) PPP5; (c) PPV3; and (d) PPV4, with screened Coulomb parameters. The scales for the intensity are different in different cases. A line width of $0.15 \mathrm{eV}$ was assumed in all cases.

experimental PA bands thus cannot be expected. The energies and the relative intensities of the theoretical induced absorptions are therefore for semiquantitative comparisons only.

In all cases in Fig. 2, the theoretical PA feature I corresponds to the $2 \mathrm{Ag}_{\mathrm{g}}$. The close proximity of this state to the $1 \mathrm{~B}_{\mathrm{u}}$ suggests that this state is outside the wavelength region within which experimental PA is observed. From Fig. 2(c) and (d), it can be concluded that PA1 in PPV derivatives is due to the calculated spectral feature II which peaks slightly below $0.4 \times E\left(1 \mathrm{~B}_{\mathrm{u}}\right)$, which would correspond to about $0.9 \mathrm{eV}$ in long chain PPV derivatives (with $E\left(1 \mathrm{~B}_{\mathrm{u}}\right) \sim$ $2.2 \mathrm{eV}$ ). This is quite close to experimental PA1 energy in PPV derivatives $[8,9]$. We remark on the PA energies in PPP derivatives in the next section, where more detailed comparisons to experiments are made.

For detailed understanding, in Table 2 we have described the wavefunctions of each of the final $\mathrm{A}_{\mathrm{g}}$ states corresponding to each band in the calculated PA spectra. Each entry in Table 2 corresponds to the overall contribution of each kind of an excitation to the many-body wave function of the excited state being considered. Remarkably, the wavefunction descriptions of the different spectral features are very similar for all four systems shown in Fig. 2. Broadly speaking, there occur three distinct classes of $A_{g}$ states in all four systems. These are discussed below.

The first class of states is represented by the $2 \mathrm{~A}_{\mathrm{g}}$, which in all cases is predominantly a superposition of even parity $d_{1} \rightarrow d_{1}^{*}$ single excitations and $\left(d_{1} \rightarrow d_{1}^{*}\right)^{2}$ double excitations. This is the standard description of the correlated $2 \mathrm{~A}_{\mathrm{g}}$ in linear chain systems. The relative weight of the single excitations is larger in the PPP oligomers than in the PPV oligomers. This is to be expected, based on the larger one-electron gap in PPP. The $2 \mathrm{~A}_{\mathrm{g}}$ can certainly be descibed within an effective linear chain model with large 
Table 2

Relative weights of the dominant contributions to the excited states of different oligomers of PPP and PPV, computed with the screened Coulomb parameters (see text)

\begin{tabular}{|c|c|c|c|c|c|c|c|}
\hline Oligmer & PA feature & State & $d_{1} \rightarrow d_{1}^{*}$ & $d_{1} \rightarrow d_{2}^{*}$ & $d_{1} \rightarrow d_{3}^{*}$ & $\left(d_{1} \rightarrow d_{1}^{*}\right)^{2}$ & $d_{1} \rightarrow l^{*} ; l \rightarrow d_{1}^{*}$ \\
\hline PPP4 & $\begin{array}{l}\text { I } \\
\text { II } \\
\text { III } \\
\text { IV }\end{array}$ & $\begin{array}{r}2 \mathrm{~A}_{\mathrm{g}} \\
3 \mathrm{~A}_{\mathrm{g}} \\
4 \mathrm{~A}_{\mathrm{g}} \\
5 \mathrm{~A}_{\mathrm{g}} \\
8 \mathrm{~A}_{\mathrm{g}} \\
10 \mathrm{~A}_{\mathrm{g}}\end{array}$ & $\begin{array}{l}0.5546 \\
0.0471 \\
0.0779 \\
0.0385 \\
- \\
-\end{array}$ & $\begin{array}{l}0.0387 \\
0.3396 \\
0.1787 \\
0.2474 \\
0.3476 \\
0.1848\end{array}$ & $\begin{array}{l}- \\
- \\
- \\
- \\
- \\
-\end{array}$ & $\begin{array}{l}0.1121 \\
0.2164 \\
0.2997 \\
0.1037 \\
0.1566 \\
0.0894\end{array}$ & $\begin{array}{l}- \\
- \\
0.0681 \\
0.0210 \\
0.0147 \\
0.3289\end{array}$ \\
\hline PPP5 & $\begin{array}{l}\text { I } \\
\text { II } \\
\text { III } \\
\text { IV }\end{array}$ & $\begin{array}{l}2 A_{g} \\
4 A_{g} \\
7 A_{g} \\
9 A_{g}\end{array}$ & $\begin{array}{l}0.6171 \\
0.1236 \\
0.1309 \\
-\end{array}$ & $\begin{array}{l}- \\
- \\
- \\
0.2284\end{array}$ & $\begin{array}{l}- \\
- \\
- \\
-\end{array}$ & $\begin{array}{l}0.0903 \\
0.4688 \\
0.4390 \\
0.0543\end{array}$ & $\begin{array}{l}- \\
0.0695 \\
- \\
0.2516\end{array}$ \\
\hline PPV3 & $\begin{array}{l}\text { I } \\
\text { II } \\
\text { III } \\
\text { IV }\end{array}$ & $\begin{array}{r}2 \mathrm{~A}_{\mathrm{g}} \\
4 \mathrm{~A}_{\mathrm{g}} \\
7 \mathrm{~A}_{\mathrm{g}} \\
10 \mathrm{~A}_{\mathrm{g}}\end{array}$ & $\begin{array}{l}0.3872 \\
0.3042 \\
0.2732 \\
-\end{array}$ & $\begin{array}{l}- \\
0.0488 \\
0.1458 \\
0.0338\end{array}$ & $\begin{array}{l}- \\
- \\
- \\
0.1746\end{array}$ & $\begin{array}{l}0.2847 \\
0.3313 \\
- \\
0.0256\end{array}$ & $\begin{array}{l}- \\
0.0169 \\
- \\
0.3193\end{array}$ \\
\hline PPV4 & $\begin{array}{l}\text { I } \\
\text { II } \\
\text { III } \\
\\
\text { IV } \\
\text { V }\end{array}$ & $\begin{array}{r}2 \mathrm{~A}_{\mathrm{g}} \\
3 \mathrm{~A}_{\mathrm{g}} \\
4 \mathrm{~A}_{\mathrm{g}} \\
5 \mathrm{~A}_{\mathrm{g}} \\
9 \mathrm{~A}_{\mathrm{g}} \\
12 \mathrm{~A}_{\mathrm{g}}\end{array}$ & $\begin{array}{l}0.5078 \\
0.1983 \\
0.4178 \\
0.0149 \\
0.0602 \\
-\end{array}$ & $\begin{array}{l}- \\
- \\
- \\
0.3366 \\
0.4555 \\
0.1140\end{array}$ & $\begin{array}{l}- \\
- \\
- \\
- \\
- \\
0.1460\end{array}$ & $\begin{array}{l}0.1884 \\
0.4680 \\
0.2483 \\
0.2859 \\
0.0318 \\
0.02071\end{array}$ & $\begin{array}{l}- \\
- \\
- \\
- \\
0.0398 \\
0.2284\end{array}$ \\
\hline
\end{tabular}

Note that the $d_{3}$ band does not occur in PPP.

dimerization that retains only the $d_{1}$ and $d_{1}^{*}$ bands, as suggested before [40].

The second class of states are represented in all cases by the different $\mathrm{A}_{\mathrm{g}}$ states that form the final states in the second and third bands in the calculated PA spectra. These have strong contributions from $\left(d_{1} \rightarrow d_{1}^{*}\right)^{2}$ double excitations, and weak but nonzero contributions from $\left(d_{1} \rightarrow l^{*} ; l \rightarrow\right.$ $\left.d_{1}^{*}\right)$. In addition, there occur also singly excited contributions of the type $d_{1} \rightarrow d_{2}^{*}, d_{2} \rightarrow d_{1}^{*}$, etc. and while there are subtle differences in the relative contributions by different kinds of single excitations involving low and high energy delocalized bands, the overall natures of the excitations that are the final states in absorption bands II and III are similar. The qualitative natures of these eigenstates are very similar to that of the $\mathrm{mA}_{\mathrm{g}}$ discussed in the context of nonlinear spectroscopy of polyacetylenes and polydiacetylenes. The wavefunction descriptions make it clear that very qualitatively these wavefunctions can also be described within the effective linear chain model [40], but with less precision than the $2 \mathrm{Ag}_{\mathrm{g}}$. Our calculated relative intensities of the $2 \mathrm{~A}_{\mathrm{g}}$ and the $\mathrm{mA}_{\mathrm{g}}$ are in qualitative agreement with other recent calculations for polyphenylenes that used a basis space of only the $d_{1}$ and $d_{1}^{*}$ bands $[41,42]$.

Above band III in the calculated spectra we always have a state that is qualitatively different from lower energy $A_{g}$ states. This corresponds to the spectral feature IV in PPP4, PPP5 and PPV3, and the spectral feature V in PPV4. Both the singly excited and doubly excited components of this state are different from those in the $\mathrm{mA}_{\mathrm{g}}$. The dominant singly excited components in the highest energy two-photon states of Fig. 2 are of the type $d_{1} \rightarrow d_{2}^{*}$ (in PPP oligomers) and $d_{1} \rightarrow d_{3}^{*}$ (in PPV oligomers). The large contribution by the outermost bands strongly suggests that the tendency of charge separation is stronger in the $\mathrm{kAg}_{\mathrm{g}}$ than in the $\mathrm{mA}_{\mathrm{g}}$. The nature of the dominant double excitations also suggest the same. The relative weights of the two classes of double excitations, $\left(d_{1} \rightarrow d_{1}^{*}\right)^{2}$ and $\left(d_{1} \rightarrow l^{*} ; l \rightarrow d_{1}^{*}\right)$, are reversed in this high energy state, with the former now making weak contribution and the latter a strong contribution. This qualitative difference between the $\mathrm{A}_{\mathrm{g}}$ states at different energies is in agreement with the earlier conjecture by Chakrabarti and Mazumdar [28]. The clear demarcation between $\mathrm{A}_{\mathrm{g}}$ states dominated by either $\left(d_{1} \rightarrow d_{1}^{*}\right)^{2}$ or $\left(d_{1} \rightarrow\right.$ $\left.l^{*} ; l \rightarrow d_{1}^{*}\right)$, rather than nearly equal admixing, is a new result. This is an effect of increased oligomer length: the energy difference between $d_{1} \rightarrow l^{*}$ and $d_{1} \rightarrow d_{1}^{*}$ excitations increases with chain length since only the delocalized MOs participate in band formation. What is also significant is the weak role of $\left(l \rightarrow l^{*}\right)^{2}$ double excitations in the $\mathrm{A}_{\mathrm{g}}$ states included in Table 2, in contrast to their relatively strong role in the high energy $A_{g}$ states of biphenyl and triphenyl within the previous limited basis calculations [28]. This is also an effect of increased chain length, with the $\left(l \rightarrow l^{*}\right)^{2}$ double excitations now perhaps dominating even higher energy distinct two-photon states. The weaker oscillator strength of absorption band IV, relative to the strong oscillator strength of absorption band II is easily understood from Table 2: double excitations of the type $\left(d_{1} \rightarrow l^{*} ; l \rightarrow d_{1}^{*}\right)$ have small dipole coupling with the $1 \mathrm{~B}_{\mathrm{u}}$, which is predominantly $d_{1} \rightarrow$ $d_{1}^{*}$. Thus the dipole coupling of the $\mathrm{A}_{\mathrm{g}}$ states responsible for absorption band IV originates mostly from the small one-excitation contribution to this state. 


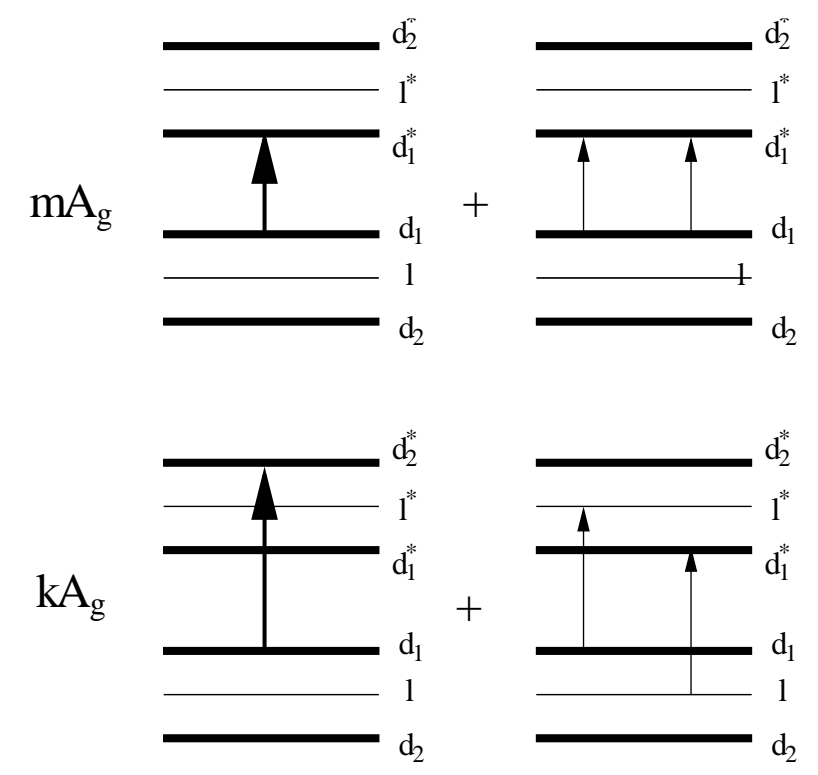

Fig. 3. Schematic representations of the $\mathrm{mA}_{\mathrm{g}}$ and $\mathrm{kA}_{\mathrm{g}}$ states for PPP. Thicker horizontal lines imply bands with finite widths. The $\mathrm{mA}_{\mathrm{g}}$ is a superposition of singly- and doubly-excited configurations both of which involve predominantly $d_{1}$ and $d_{1}^{*}$ bands only. The one-excitation component of $\mathrm{kA}_{\mathrm{g}}$ involves $d_{1} \rightarrow d_{2}^{*}$ excitations in PPP ( $d_{1} \rightarrow d_{3}^{*}$ in PPV), while the two-excitation components involve the $l$ and $l^{*}$ bands. The thick arrows denote single excitations with $\mathrm{A}_{\mathrm{g}}$ symmetry reached by two successive dipole-allowed transitions. Wherever applicable, particle-hole reversed excitations are also implied.

Taken together, the results of Table 2 indicate the occurrence of different classes of $\mathrm{A}_{\mathrm{g}}$ states, which are distinguished by the dominance of different types of one- and two-excitations as depicted schematically in Fig. 3. With hindsight, this is perhaps not entirely surprising, as discussed in Section 3.

\section{Conclusions and discussions}

Even as the concept of different types of two-photon states appear to be correct, it might seem that straightforward assignments of experimental PA1 and PA2 from the calculated PA spectra of Fig. 2 alone are not possible. We first discuss applications of our theory to PPV derivatives, and then to ladder PPP, etc. The strong spectral feature II in the theoretical spectra (the $\mathrm{mA}_{\mathrm{g}}$ ) is certainly a component of the experimental PA1 in PPV derivatives. Beyond this there are two possibilities, viz. (i) the spectral feature III corresponds to PA2, and the spectral feature IV (V in PPV4) is too high in energy to be observed experimentally; or (ii) the spectral feature III is also a part of PA1 (especially in long chains) and it is the high energy excitation that is a superposition of $d_{1} \rightarrow$ $d_{2}^{*}\left(d_{1} \rightarrow d_{3}^{*}\right)$ and $\left(d_{1} \rightarrow l^{*} ; l \rightarrow d_{1}^{*}\right)$ that corresponds to PA2. We ascribe the latter excitation to PA2 based on the following reasons. First, $\mathrm{A}_{\mathrm{g}}$ states that give rise to spectral feature III occur also in linear chain polyenes [20]. Their contribution to EA and TPA are vanishingly weak in long chains. In contrast, the contributions of the experimental $\mathrm{kA}_{\mathrm{g}}$ to EA and TPA in PPV derivatives are clearly visible $[9,14]$. On the other hand, eigenstates that are predominantly $d_{1} \rightarrow$ $d_{2}^{*}+\left(d_{1} \rightarrow l^{*}+l \rightarrow d_{1}^{*}\right)$ are clearly absent in the polyacetylenes and polydiacetylenes, which possess only one delocalized valence and conduction band each, and hence our assignment would naturally explain the absence of the $\mathrm{kA}_{\mathrm{g}}$ state in these systems [14]. Second, spectral feature III (but not IV) has also been found in the calculations by Beljonne [42], who, however, determined that in the long chain limit the energies of features II and III converge. This once again supports our assignment of the highest energy two-photon excitation in Fig. 2 to PA2. We have already pointed out that the calculated PA1 energy is reasonably close to the experimental PA1 energy in PPV derivatives. The calculated scaled energy of PA2 from Fig. 2(c) and (d), at $0.7-0.8 \times E\left(1 \mathrm{~B}_{\mathrm{u}}\right)$ (again with experimental $E\left(1 \mathrm{~B}_{\mathrm{u}}\right)=2.2 \mathrm{eV}$ in substituted PPVs) is at $1.5-1.76 \mathrm{eV}$, which is a reasonably good fit to the experimental PA2 energy of $1.3-1.4 \mathrm{eV}$ in PPV derivatives, given the very short lengths of our oligomers, and the increased difficulty of fitting of very high energy states.

We believe that the recent experiments on a methylsubstituted ladder type PPP ( $m$-LPPP) [12] and on a ladder type oligophenyl [13] have probed an energy region that is considerably above the region where the $\mathrm{mA}_{\mathrm{g}}$ occurs. The PA features that have been called PA1 in these works occur at $1.5 \mathrm{eV}$ in the polymer and at $1.8 \mathrm{eV}$ in the oligomer, and are therefore too high in energy to be the same as PA1 in PPV derivatives. We therefore believe that the observed lowest energy PA features actually correspond to PA2 of Frolov et al., and this is why charge separation occurs upon excitation to this energy. This assignment is supported by the observation of EA [43] as well as TPA [44] to a different lower energy two-photon state that occurs $\sim 0.7 \mathrm{eV}$ above the $1 \mathrm{~B}_{\mathrm{u}}$ exciton in the polymer. We therefore make the testable prediction that there should occur in these systems a lower energy PA at $\sim 0.7 \mathrm{eV}$ that is considerably stronger than the PAs in the $1.5-1.8 \mathrm{eV}$ range. The scaled PA2 energy from our PPP oligomer calculations, 0.6-0.8 $\times E\left(1 \mathrm{~B}_{\mathrm{u}}\right)$, with experimental $E\left(1 \mathrm{~B}_{\mathrm{u}}\right)=2.7 \mathrm{eV}$ in $m$-LPPP [43], corresponds to $1.6-2.1 \mathrm{eV}$, once again reasonably close to the experimental value.

The above then leads to the question why dissociation of the $\mathrm{kA}_{\mathrm{g}}$ to polaron pair is so efficient. We speculate that this is related to the specific structure of the $\mathrm{kAg}_{\mathrm{g}}$. To begin with, as already mentioned, the binding energy of an exciton whose one-excitation component involves the outermost bands is necessarily weak. Similar conclusion can also be obtained regarding the two-excitation component, based on earlier work. In photoconductivity measurement on a PPV derivative it has been found that a large jump in the photoconductivity occurs at $4.7 \mathrm{eV}$, exactly where the $d_{1} \rightarrow l^{*}+l \rightarrow d_{1}^{*}$ one-photon exciton is located [29]. The jump in the photoconductivity is due to a sudden increase in the interchain charge carrier generation subsequent to the excitation to this particular excited state. We speculate that 
there occurs a similar enhanced charge carrier generation subsequent to the sequential excitation to an $\mathrm{A}_{\mathrm{g}}$ state that has strong contributions from $\left(d_{1} \rightarrow l^{*} ; l \rightarrow d_{1}^{*}\right.$ ) (whose energy is however lower and close to the minus combination of the one-excitations). Indeed, a similar mechanism for charge generation has also been suggested by Zenz et al. [45]. In the original work by Köhler et al., theoretical calculations suggested that the enhanced dissociation of the $d_{1} \rightarrow$ $l^{*}+l \rightarrow d_{1}^{*}$ state was a consequence of the highly delocalized electron-hole character of this state [29]. The latter necessarily implies that the charge carriers are similarly weakly bound even in the double excitation $\left(d_{1} \rightarrow l^{*} ; l \rightarrow d_{1}^{*}\right)$. An additional reason for the violation of the Vavilov-Kasha rule upon excitation to the $\mathrm{kAg}_{\mathrm{g}}$ can be as follows. There exists a fundamental similarity between the $1 \mathrm{~B}_{\mathrm{u}}$ and the $\mathrm{mA}_{\mathrm{g}}$ in that both are composed of predominantly $d_{1}$ and $d_{1}^{*}$ MOs (of, however, opposite spatial symmetries). The $\pi$-electron bond orders in these states are therefore similar, and the energy minima on the potential energy surface should be close to one another. In contrast, the bond orders of states involving the localized MOs with zero $\pi$-electron densities on certain carbon atoms are necessarily different, and it is conceivable that the energy minimum of the $\mathrm{kA}_{\mathrm{g}}$ occurs at a very different region of the potential energy surface. This can prevent efficient relaxation to the $1 \mathrm{~B}_{\mathrm{u}}$, and given the small exciton binding energy, charge separation can now occur. Further theoretical work is being pursued along these direction.

\section{Acknowledgements}

Our work on the polyphenylenes have been heavily influenced by the original work on these materials by Michael Rice and collaborators, who were the first to make the symmetry characteristics of the different kinds of single excitations in polyphenylenes clear. As with many other topics in the area of organic solids, Michael was a pioneer in this area. Work at Arizona was partially supported by NSF DMR-0101659, NSF ECS-0108696, and the ONR. We acknowledge many useful discussions with G. Lanzani and Z.V. Vardeny.

\section{References}

[1] M. Chandross, S. Mazumdar, M. Liess, P.A. Lane, Z.V. Vardeny, M. Hamaguchi, K. Yoshino, Phys. Rev. B 55 (1997) 1486.

[2] M.J. Rice, Yu.N. Gartstein, Phys. Rev. Lett. 73 (1994) 2504.

[3] Y. Shimoi, S. Abe, Synth. Met. 78 (1996) 219.

[4] D. Comoretto, G. Dellepiane, F. Marabelli, J. Cornil, D. do Santos, J. Brédas, D. Moses, Phys. Rev. B 62 (2000) 10173.

[5] M. Yan, L.J. Rothberg, F. Papadimitrakopoulos, M.E. Galvin, T.M. Miller, Phys. Rev. Lett. 72 (1994) 1104.

[6] J. Hsu, M. Yan, T.M. Jedju, L.J. Rothberg, B.R. Hsieh, Phys. Rev. B 49 (1994) 712.

[7] J. Leng, S. Jeglinski, X. Wei, R.E. Benner, Z.V. Vardeny, F. Guo, S. Mazumdar, Phys. Rev. Lett. 72 (1994) 156.
[8] S.V. Frolov, Z. Bao, M. Wohlgenannt, Z.V. Vardeny, Phys. Rev. Lett. 85 (2000) 2196.

[9] S.V. Frolov, Z. Bao, M. Wohlgenannt, Z.V. Vardeny, Phys. Rev. B 65 (2001) 205209.

[10] V.I. Klimov, D.W. McBranch, N. Barashkov, J. Ferraris, Phys. Rev. B 58 (1998) 7654.

[11] B. Kraabel, V.I. Klimov, R. Kohlman, S. Xu, H.L. Wang, D.W. McBranch, Phys. Rev. B 61 (2000) 8501.

[12] C. Gadermaier, G. Cerullo, G. Sansone, G. Leising, U. Scherf, G. Lanzani, Phys. Rev. Lett. 89 (2002) 117402.

[13] C. Gadermaier, G. Cerullo, M. Zavelani-Rossi, G. Sansone, G. Lanzani, E. Zojer, A. Pogantsch, D. Beljonne, Z. Shuai, J.L. Brédas, et al., Phys. Rev. B 66 (2002) 125203.

[14] M. Liess, S. Jeglinsky, Z.V. Vardeny, M. Ozaki, K. Yoshino, Y. Ding, T. Barton, Phys. Rev. B 56 (1997) 15712.

[15] S.J. Martin, D.D.C. Bradley, P.A. Lane, H. Mellor, P.L. Burn, Phys. Rev. B 59 (1999) 15133.

[16] A. Mathy, K. Ueberhofen, R. Schenk, H. Gregorius, R. Garay, K. Müllen, C. Bubeck, Phys. Rev. B 53 (1996) 4367.

[17] C.J. Baker, O.M. Gelsen, D.D.C. Bradley, Chem. Phys. Lett. 201 (1993) 127.

[18] U. Lemmer, R. Fischer, J. Feldmann, R.F. Mahrt, J. Yang, A. Greiner, H. Bässler, E. Göbel, H. Heesel, H. Kurz, Chem. Phys. Lett. 203 (1993) 28.

[19] S.N. Dixit, D. Guo, S. Mazumdar, Phys. Rev. B 43 (1991) 6781.

[20] M. Chandross, Y. Shimoi, S. Mazumdar, Phys. Rev. B 59 (1999) 4822.

[21] P.C.M. McWilliams, G.W. Hayden, Z.G. Soos, Phys. Rev. B 43 (1991) 9777.

[22] Z.G. Soos, R.G. Kepler, Phys. Rev. B 43 (1991) 11908.

[23] S. Abe, M. Schreiber, W.P. Su, J. Yu, Phys. Rev. B 45 (1992) 9432.

[24] D. Beljonne, R. Cornil, Z. Shuai, J. Brédas, F. Rohlfing, D.D.C. Bradley, W.E. Torruellas, V. Ricci, G.I. Stegeman, Phys. Rev. B 55 (1997) 1505.

[25] D. Yaron, Phys. Rev. B 54 (1996) 4609.

[26] A. Race, W. Barford, R.J. Bursill, Phys. Rev. B 64 (2001) 035208.

[27] J.B. Birks, Photophysics of Aromatic Molecules, Wiley/Interscience, London, 1970.

[28] A. Chakrabarti, S. Mazumdar, Phys. Rev. B 59 (1999) 4839.

[29] A. Köhler, D.A. dos Santos, D. Beljonne, Z. Shuai, J.L. Brédas, A.B. Holmes, A. Kraus, K. Müllen, R.H. Friend, Nature (London) 392 (1998) 903.

[30] K. Ohno, Theor. Chim. Acta 2 (1964) 219.

[31] C.W.M. Castleton, W. Barford, J. Chem. Phys. 117 (2002) 3570.

[32] Z.V. Vardeny, private communications.

[33] P. Tavan, K. Schulten, Phys. Rev. B 36 (1987) 4337.

[34] H. Ghosh, A. Shukla, S. Mazumdar, Phys. Rev. B 62 (2000) 12763.

[35] A. Shukla, Phys. Rev. B 65 (2002) 125204.

[36] M. Chandross, S. Mazumdar, S. Jeglinski, X. Wei, Z.V. Vardeny, E.W. Kwock, T.M. Miller, Phys. Rev. B 50 (1994) 14702.

[37] E.K. Miller, D. Yoshida, C.Y. Yang, A.J. Heeger, Phys. Rev. B 59 (1999) 4661.

[38] A. Shukla, H. Ghosh, S. Mazumdar, Phys. Rev. B 67 (2003) 245203.

[39] S. Mazumdar, D. Guo, S.N. Dixit, J. Chem. Phys. 96 (1992) 6862.

[40] Z.G. Soos, S. Etemad, D.S. Galvao, S. Ramasesha, Chem. Phys. Lett. 194 (1992) 341.

[41] M.Y. Lavrentiev, W. Barford, S.J. Martin, H. Daly, R.J. Bursill, Phys. Rev. B 59 (1999) 9987.

[42] D. Beljonne, Habilitation Thesis, Universite de Mons-Hainaut, Belgium, 2001.

[43] M.G. Harrison, S. Moller, G. Weiser, G. Urbasch, R.F. Mahrt, H. Bässler, U. Scherf, Phys. Rev. B 60 (1999) 8650.

[44] M.G. Harrison, G. Urbasch, R.F. Mahrt, H. Giessen, H. Bässler, U. Scherf, Chem. Phys. Lett. 313 (1999) 755.

[45] C. Zenz, G. Lanzani, G. Cerullo, W. Graupner, G. Leising, U. Scherf, S. DeSilvestri, Synth. Met. 116 (2001) 27. 